\title{
Parameter configuration of composite vibration isolation system using power flow and MOPSO technique
}

\author{
Huang Wei ${ }^{1,3}$, Xu Jian ${ }^{2}$, Zhu Da-Yong ${ }^{1,3, a}$, Hu Ming-Yi ${ }^{4,5}$, Lu Jian-Wei ${ }^{6}$, \\ Lu Kun-Lin ${ }^{1,3}$ AND WANG JUn-CAI ${ }^{1,3}$ \\ 1 School of Civil and Hydraulic Engineering, Hefei University of Technology, Hefei, P.R. China \\ 2 China National Machinery Industry Corporation, Beijing, P.R. China \\ 3 Anhui Provincial Laboratory of Civil Engineering And Materials, Hefei, P.R. China \\ 4 China Electronics Engineering Design Institute, Beijing 100142, P.R. China \\ ${ }^{5}$ Department of Civil Engineering, Tsinghua University, Beijing 100084, P.R. China \\ ${ }^{6}$ School of Mechanical and Automotive Engineering, Hefei University of Technology, Hefei, P.R. China
}

Received 16 July 2015, Accepted 14 November 2015

\begin{abstract}
Traditional vibration isolation design often aims to suppress vibrations from machinery equipment, while vibrations in the foundation are usually ignored. In this paper, the "machinery equipment single-stage (double-stage) isolators - thin plate foundation" is considered as a composite vibration isolation system, with equipment deployed at four positions on the plate. The forces transmitted from the equipment to the foundation were derived using a mechanical four-pole connection principle, and the displacement admittance of the junctions between the equipment and the plate was used as the key intermediate variable. Multi-objective optimization for the proposed system was subsequently performed, using fitness functions to reduce the maximum power flow transmitted to the plate and ensure a uniform vibration of the machinery equipment. A multi-objective particle swarm optimization (MOPSO) algorithm was used as the optimization tool which offers the advantages of fewer parameter settings, fast convergence, a strong optimization capability, and possession of a unique, global and optimal solution (gbest solution) based on Pareto dominance. The power flow that induced plate vibrations from the machinery isolation system was introduced in this study, which was used to consider the transmitted force and velocity on the plate simultaneously. An advanced theory of clamped plate vibration aptly supported this strategy, and inspiration was also provided by an innovative optimization strategy based on artificial intelligence.
\end{abstract}

Key words: Machinery equipment / single-stage / double-stage / clamped thin plate / four positions installation / composite vibration isolation system / admittance / power flow / uniform vibration / multi-objective particle swarm optimization

\section{Introduction}

In modern manufacturing and industrial engineering, many types of machinery equipment are commonly used in engineering applications, including machines for rotating, reciprocating, impacting and other typical functions. Advances in science and technology have gradually prompted equipment to move towards the high-tech era. Vibration is a key problem when utilizing machinery equipment, and there has been significant work done to isolate and attenuate any harmful vibrations in the equipment from the surrounding environment.

In traditional studies, the foundation is generally regarded as completely rigid, so its vibration is not taken

\footnotetext{
a Corresponding author: zhudymeng@163.com
}

into account. However, when there is a high-frequency external stimulus and the foundation stiffness is low, traditional strategies of vibration isolation will not achieve the desired effects, and even the dynamic performance will deteriorate seriously. Therefore, the vibration of the foundation must be involved in the strategy used. A plate is commonly used as the foundation in machinery equipment, which for computational simplicity is generally viewed as being supported by a simple structure. However, the clamped form is used for a wide range of applications, such as the floors of industrial buildings, and thus it cannot be ignored. Amabili et al. [1] performed a study on large-amplitude forced vibrations of a clamped plate excited by a centralized harmonic force. Another study [2] conducted a comparative study of active and 


\section{Nomenclature}

\begin{tabular}{|c|c|}
\hline$\vec{F} \mathrm{e}^{i \omega t}$ & External force on the structure \\
\hline$\vec{V} \mathrm{e}^{i \omega t}$ & Velocity response of any point on the structure \\
\hline$P$ & Power flow \\
\hline$T$ & Time period \\
\hline$\omega$ & Circular frequency \\
\hline$P L$ & Power level \\
\hline$i$ & The $i$ th particle \\
\hline$j$ & The $j$ th dimension of each particle \\
\hline$v_{i j}$ & Flight velocity vector of particles \\
\hline$x_{i j}$ & Flight displacement vector of particles \\
\hline$t$ & The $t$ th iteration \\
\hline pbest & Local optimum \\
\hline gbest & Global optimum \\
\hline$c_{1}, c_{2}$ & Acceleration factors or learning factors \\
\hline$r_{1}, r_{2}$ & Random numbers between $(0,1)$ \\
\hline$w$ & Inertia weight factor \\
\hline$X$ & Vertical displacement of any point on the plate \\
\hline$V$ & Vertical velocity of any point on the plate \\
\hline$(x, y)$ & Discrete points of the plate \\
\hline$T_{\mathrm{f}}$ & Duration of external force excited on the plate \\
\hline$\left(x^{\prime}, y^{\prime}\right)$ & Acting point of external force on the plate \\
\hline$M, N$ & Modal numbers \\
\hline$\Psi(\bullet)$ & Modal function \\
\hline$B$ & Bending stiffness of the plate \\
\hline E & Young's modulus \\
\hline$v$ & Poisson's ratio \\
\hline$\rho$ & Volume density of the plate \\
\hline$\rho_{s}$ & Surface density of the plate \\
\hline$\omega_{M N}$ & Natural frequencies of the plate \\
\hline$a, b, h$ & Length, width and height of the plate \\
\hline$k, c$ & Stiffness and damping coefficients of the isolators (single-stage) \\
\hline$m$ & Mass of the machinery equipment (single-stage) \\
\hline$\omega_{0}, \xi$ & Natural frequency and damping ratio of the isolators (single-stage) \\
\hline$[\hat{m}]$ & Four-pole expression of the mass \\
\hline$\left[\hat{k}^{*}\right]$ & Four-pole expression of the stiffness and damping in parallel \\
\hline$T_{\mathrm{F}}$ & Force transmissibility \\
\hline$M_{\mathrm{A}}, M_{\mathrm{B}}, M_{\mathrm{C}}, M_{\mathrm{D}}$ & Admittance of the four installation points on the plate \\
\hline$F_{\mathrm{A}}, F_{\mathrm{B}}, F_{\mathrm{C}}, F_{\mathrm{D}}$ & Four transmitted forces to the plate \\
\hline $\operatorname{Peak}(\mathrm{A}), \operatorname{Peak}(\mathrm{B}), \operatorname{Peak}(\mathrm{C}), \operatorname{Peak}(\mathrm{D})$ & Peak displacements of installations on the plate, A, B, C and D \\
\hline$N_{1}, N_{2}, N_{3}$ & Proportion factors between transmitted forces \\
\hline$m_{1}$ & Mass of machinery equipment (double-stage) \\
\hline$m_{2}$ & Mass of secondary system (double-stage) \\
\hline$k_{1}, c_{1}$ & Stiffness and damping coefficients of primary system (double-stage) \\
\hline$k_{2}, c_{2}$ & Stiffness and damping coefficients of the secondary system (double-stage) \\
\hline$u, n_{1}, n_{2}, \xi_{2}, \theta$ & Derivation parameters for force transmissibility (double-stage) \\
\hline
\end{tabular}

passive vibration isolation of a clamped thin plate, which aimed to examine the piezoelectric materials in active vibration control. A free vibration problem of a clamped thin plate using a quasi-Green function was solved in reference [3]. Arenas [4] derived a general vibration expression for a clamped thin plate by means of a virtual work principle, in which it was not necessary to solve the simultaneous equations, overcoming the complicated symmetric characteristic of the Rayleigh-Ritz method.

When vibration transmissions between points on a continuous structure are studied, it is often not sufficient to rely solely on the transference of measurements to provide enough information to determine the pathway. The concept of power flow has become popular in research as an effective measure to improve vibration control. The main advantage of the power flow strategy is that it simultaneously takes force and velocity into account and also considers the impedance characteristic. Since power can be used to express the vibration as an absolute measurement of the energy transmission, reducing the power flow into the structure is quite effective in vibration isolation. The power flow behaviour and dynamics of a vibration isolation system were studied in reference [5], and the mathematical equations governing 
the dynamics characteristics were also derived. An efficient and reliable evolutionary-based approach to solve the optimal power flow problem was presented in reference [6], and some novel approaches were reviewed to obtain the optimal variables of power flow. In reference [7], an artificial bee colony algorithm based on the optimal power flow was utilized to minimize the active power loss in power systems, and it provided much inspiration on the application of swarm intelligence to power flow optimization. Govder and White [8-10] were the first to study the transference mechanism of power flow in machinery and infrastructure. Reference [11] presented detailed admittance expressions for the continuous structures, such as plates, beams, and composite structures. In order to investigate the power flow transmission of machinery equipment which has multiple points installed, the effective mobility and overall mobility were presented by another study [12]. The effective mobility described the dynamic mobility excited by a single-point installation, and the overall mobility expressed the overall mobility of all points. In reference [13], a multiple-objective genetic algorithm was applied to optimize the key parameters in the control strategy of an electric vehicle electro-hydraulic composite braking system, and the numerical results confirmed a good astringency.

Single-stage and double-stage systems are the main strategies for vibration isolation of machinery equipment, and isolation performance is closely related to parameter configuration. Appropriate selection of parameters can improve the performance, while poor parameter selection will be counterproductive. A mathematical model was derived for a suspension system which enabled detailed parameter investigation to be undertaken using gradient optimization [14]. To investigate parameter optimization of a double-stage vibration isolation system, a novel optimization strategy using a maximum entropy approach combined with a Genetic Algorithm (GA) was developed and used to optimize the parameters [15]. Huang et al. [16] performed multi-objective optimization for isolation parameters of single-stage and double-stage systems using the particle swarm optimization (PSO) technique.

In general, traditional gradient-based optimization requires the computation of sensitivity factors and eigenvectors during its iteration process. This places a heavy computational burden on the method with slow convergence. Moreover, there is no local criterion that can be used to decide whether a local solution is also the global solution. Thus, conventional optimization methods using derivatives and gradients are generally unable to locate or identify the global optimum. This may not be an issue for real-world applications, where a good solution may be sufficient, even if it is not the best solution. Consequently, heuristic methods are widely used for the global optimization problem. The PSO algorithm was first proposed by Eberhart and Kennedy [17] and has become a useful tool for engineering optimization. It is a novel populationbased metaheuristic method which uses swarm intelligence generated by cooperation and competition between the particles in a swarm.
Coello et al. [18] first proposed the multi-objective particle swarm optimization (MOPSO) algorithm. Flying particles can be guided by determining the flying direction of each particle with optimal Pareto sets. Using non-dominated solutions that have been found before and are contained in the global knowledge base, a unique, optimal and global solution can be obtained using a specific algorithm. The advantages of MOPSO have been effectively verified and include fewer parameter settings, fast convergence, a strong optimization capability, and possession of a unique, global and optimal solution gbest based on Pareto domination [19].

\section{Basic theory of vibration power flow}

\subsection{Power flow generated by a single-point harmonic excitation}

Denote $F(t)=\vec{F} \mathrm{e}^{i \omega t}$ as the force excited by the vibration infrastructure and $V(t)=\vec{V} \mathrm{e}^{i \omega t}$ as the velocity response. Then the power input to the structure can be denoted as $P=F(t) V(t)$. For any period of time $T$, the vibration power flow can be written as:

$$
P=\lim _{T \rightarrow \infty} \int_{0}^{T} F(t) V(t) \mathrm{d} t
$$

Then Equation (1) can be rewritten as:

$$
P=\frac{1}{T} \int_{0}^{T} \operatorname{Re}\left(\vec{F} \mathrm{e}^{j \omega t}\right) \operatorname{Re}\left(\vec{V} \mathrm{e}^{j \omega t}\right) \mathrm{d} t
$$

where $\vec{F}$ and $\vec{V}$ are complex vectors. Equation (2) can be simplified as:

$$
P=\frac{1}{2} \operatorname{Re}\left(\vec{F} \cdot \vec{V}^{*}\right)=\frac{1}{2} \operatorname{Re}\left(\vec{F}^{*} \cdot \vec{V}\right)
$$

where $*$ shows the complex conjugate operation.

\subsection{Power flow generated by a multi-point harmonic excitation}

When multiple excitations are forced onto the infrastructure, the overall power flow is equal to the sum of all power flows generated by each force, since power flow is scalar. The $n$ forces are $F_{1}, F_{2}, \ldots, F_{n}$ and, the induced velocity responses of each force are $V_{1}, V_{2}, \ldots, V_{n}$, thus the power flow can be denoted as:

$$
P=\frac{1}{2} \operatorname{Re}\left\{[F]^{H}[V]\right\}
$$

where $H$ shows the conjugate transpose operation.

Since power flow has a broadly changing scope, the concept of a Power Level is introduced here [20] which is expressed as:

$$
P L=10 \times \log (P / 1 e-12) \mathrm{d} B
$$




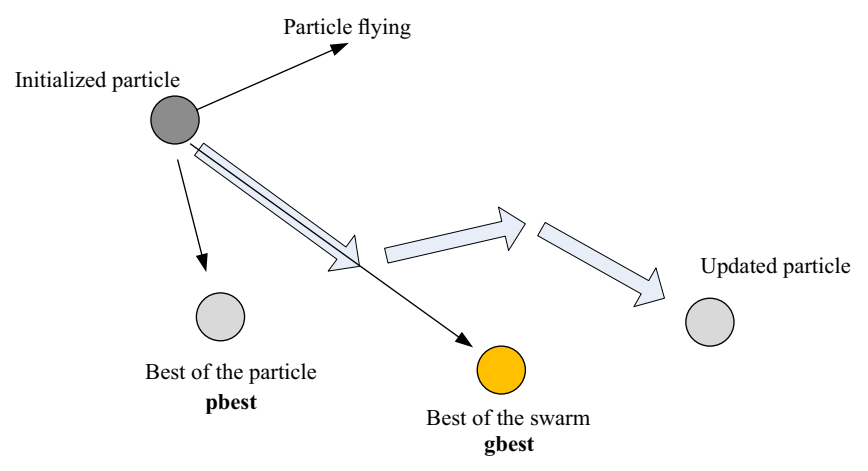

Fig. 1. Basic structure of PSO algorithm.

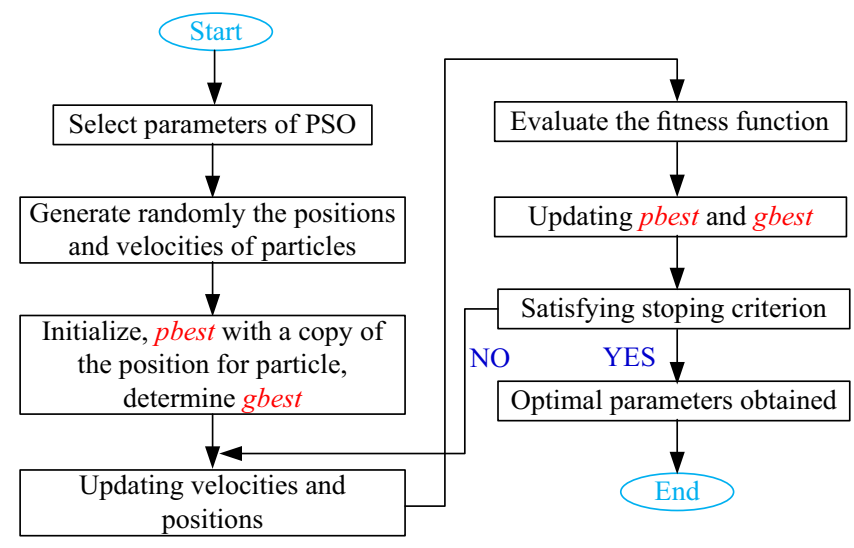

Fig. 2. Schematic diagram of PSO.

\section{MOPSO algorithm}

PSO is a type of random optimization method that can be classed as an artificial intelligence method. This algorithm was inspired by the social behavior of animals and insects such as bird flocks and fish schools. PSO employs a swarm of multiple particles, which each has its own position and velocity. All of the particles share information obtained from the other particles, and efficient searching is obtained through interaction between the particles. A basic structure of the PSO is shown in Figure 1. As shown, the direction of the particle motion is a linear combination of individual particles with random coefficients for each vector, which ultimately results in an improved particle position. In this way, a good search is performed within the space and appropriate solutions are obtained for the optimization problem. A schematic diagram of PSO is shown in Figure 2.

In the PSO algorithm, the state of each particle can be described by a group of position and velocity vectors which together represent all possible solutions and movement directions within the search space. The desired solution can be found through constantly updating the optimal neighbor solutions each time new global and optimal solutions are discovered. The main steps of MOPSO can be summarized as follows:

- Step 1: Initialize the population, and compute the corresponding objective vectors of the particles, and add the non-dominated solutions to the external archive.
- Step 2: Initialize the local optimum pbest of particles and the global optimum gbest.

- Step 3: Amend the velocities and positions of the particles by evaluating the following equations so as to generate a new pbest;

$$
\begin{aligned}
v_{i j}= & w v_{i j}(t)+c_{1} r_{1}\left(\text { pbest }_{i j}(t)-x_{i j}(t)\right) \\
& +c_{2} r_{2}\left(\text { gbest }_{i j}(t)-x_{i j}(t)\right) \\
x_{i j}(t+1)= & x_{i j}(t)+v_{i j}(t+1)
\end{aligned}
$$

where $i$ represents the $i$ th particle; $j$ represents the $j$ th dimension of each particle; $t$ represents the $t$ th iteration; $v_{i j}(t)$ represents the flight velocity vector; $x_{i j}(t)$ represents the flight displacement vector; pbest represents the optimal location component; gbest represents the optimal position of the whole swarm; $c_{1}, c_{2}$ are acceleration factors or learning factors; $r_{1}, r_{2}$ are random numbers between $(0,1)$; and $w$ is the inertia weight factor.

- Step 4: Maintain an external archive using the obtained new non-dominated solution, and select gbest for each particle (the archive determines the selection of the global optimum).

- Step 5: Determine whether the maximum iteration has yet been achieved. If not, the program will continue; if it has, terminate the computation, and output the optimal Pareto solution set and gbest.

It is important to point out that when a traditional multiobjective optimization is utilized, a set of equivalent solutions will be generated, and it is difficult to determine which is the desired solution. Pareto dominating is the most direct method of solving this problem, since it considers all of the non-dominated solutions in the archive, and then determines a "leader". The density measuring technique is commonly used to determine the global optimum. The nearest neighbor density estimation (NNDE) method [21], which is based on nearest neighbor congestion evaluation of particles is adopted in this paper. In the NNDE method, the crowding distance can be measured by a cuboid which consists of neighboring particles, and a longer perimeter may indicate a lower density distribution of individuals and a better adaptability. A schematic diagram of the NNDE method is depicted in Figure 3. There are certainly other similar methods that could alternatively be used, such as the kernel density estimation method [22].

\section{Vibration of clamped thin plate}

Arenas proposed an analytical form of the clamped thin plate vibration forced by harmonic excitation, which is given by:

$X(x, y)=F_{0} \sum_{M=1}^{\infty} \sum_{N=1}^{\infty} \frac{\Psi_{M N}(x, y) \Psi_{M N}\left(x^{\prime}, y^{\prime}\right)}{B\left(I_{1} I_{2}+2 I_{3} I_{4}+I_{5} I_{6}\right)-\rho_{s} \omega^{2} I_{2} I_{6}}$

where $F_{0}$ is the force amplitude; $B=E h^{3} /\left[12\left(1-v^{2}\right)\right]$ is the bending stiffness of the thin plate; $E$ is Young's modulus; $v$ is Poisson's ratio; $\rho_{s}=\rho h$ is the surface density 


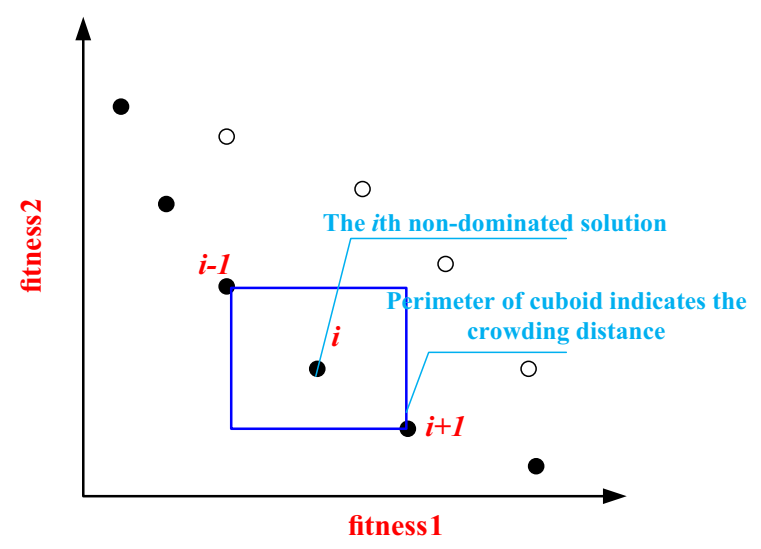

Fig. 3. Schematic diagram of the nearest neighbor density estimation method.

of the plate; $\rho$ is the volume density of the plate; $(x, y)$ is a discrete point on the thin plate and $\left(x^{\prime}, y^{\prime}\right)$ is the point that the exciting force acts on the plate.

The modal function $\Psi_{M N}(x, y)$ can be expressed as:

$$
\Psi_{M N}(x, y)=\theta_{M}(x) \zeta_{N}(y)
$$

$$
\begin{aligned}
\theta_{M}(x) & =J\left(\frac{\beta_{M} x}{a}\right)-\frac{J\left(\beta_{M}\right)}{H\left(\beta_{M}\right)} H\left(\frac{\beta_{M} x}{a}\right), \\
\zeta_{N}(y) & =J\left(\frac{\beta_{N} y}{b}\right)-\frac{J\left(\beta_{N}\right)}{H\left(\beta_{N}\right)} H\left(\frac{\beta_{N} y}{b}\right) ; \\
J(\bullet) & =\cosh (\bullet)-\cos (\bullet), H(\bullet)=\sinh (\bullet)-\sin (\bullet), \\
& \text { and } \beta_{i} \text { is the root of } \cosh (\beta) \cos (\beta)=1 ; \\
I_{2} I_{6} & =\frac{a b}{\beta_{M} \beta_{N}} L_{M} L_{N} ; I_{3} I_{4}=\frac{\beta_{M} \beta_{N}}{a b} R_{M} R_{N} ; \\
I_{1} & =I_{6}\left(\frac{\beta_{M}}{a}\right)^{4} ; I_{5}=I_{2}\left(\frac{\beta_{N}}{b}\right)^{4} ; \\
L_{i} & =\frac{\left(1+D_{i}^{2}\right) \sinh \left(2 \beta_{i}\right)}{4}+\sinh \left(\beta_{i}\right)\left[2 D_{i} \sin \left(\beta_{i}\right)\right. \\
& \left.-\left(1-D_{i}^{2}\right) \cos \left(\beta_{i}\right)\right]-\left(1+D_{i}^{2}\right) \sin \left(\beta_{i}\right) \cosh \left(\beta_{i}\right) \\
& +\left(1-D_{i}^{2}\right) \sin \left(\beta_{i}\right) \cos \left(\beta_{i}\right)+\beta_{i} \\
& -\frac{D_{i}\left[1+\cosh \left(2 \beta_{i}\right)\right]}{2}+D_{i} \cos ^{2}\left(\beta_{i}\right) ; \\
R_{i}= & \frac{\left(1+D_{i}\right)^{2} \sinh \left(2 \beta_{i}\right)}{4}-\frac{D_{i} \cosh \left(2 \beta_{i}\right)}{2} \\
& -\frac{\left(1-D_{i}^{2}\right) \sin \left(\beta_{i}\right) \cos \left(\beta_{i}\right)}{2}-D_{i} \cos ^{2}\left(\beta_{i}\right) \\
- & D_{i}^{2} \beta_{i}+\frac{3 D_{i}}{2} ; D_{i}=\frac{J\left(\beta_{i}\right)}{H\left(\beta_{i}\right)} .
\end{aligned}
$$

In practice, the infinite series in Equations (8) and (9) will be truncated to finite terms. $M, N=1-6$ is adopted in this study.

The natural frequencies of a rectangular clamped plate are given by:

$\omega_{M N}=\sqrt{\frac{B}{\rho_{s}}} \sqrt{\left(\frac{\beta_{M}}{a}\right)^{4}+\left(\frac{\beta_{N}}{b}\right)^{4}+2\left(\frac{\beta_{M} \beta_{N}}{a b}\right)^{2} \frac{R_{M} R_{N}}{L_{M} L_{N}}}$

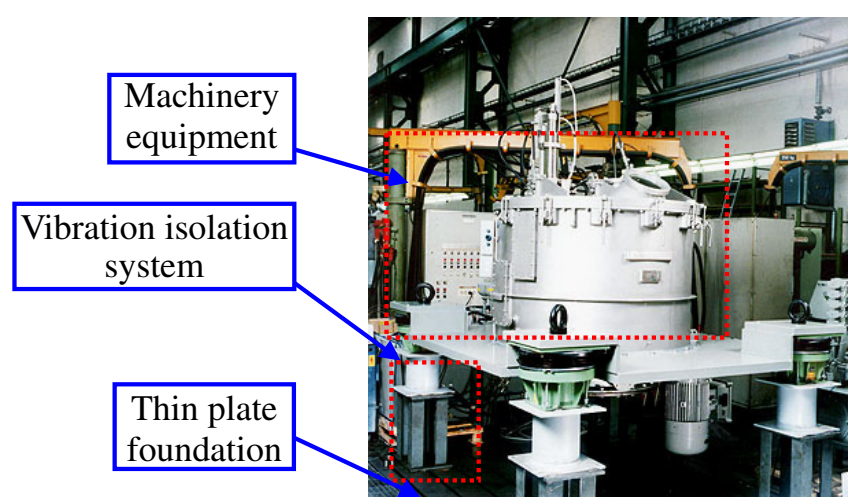

Fig. 4. Typical machinery equipment.

For a multi-point force excitation (several forces of amplitude $F_{1}, F_{2}, \ldots, F_{k}$ applied to a set of corresponding coordinates $\left(x_{1}, y_{1}\right),\left(x_{2}, y_{2}\right), \ldots,\left(x_{k}, y_{k}\right)$ on the plate), the total displacement of the plate is obtained using superposition of the responses induced by each of the forces, since the system is assumed to be linear. The total displacement, including the response of modes up to the mode $(M, N)$, is then:

$$
X(x, y)=\frac{1}{\rho_{s} a b} \sum_{i=1}^{k} \sum_{M=1}^{\infty} \sum_{N=1}^{\infty} \frac{\Psi_{M N}(x, y) \Psi_{M N}\left(x^{\prime}, y^{\prime}\right)}{\gamma\left(\omega_{M N}^{2}-\omega^{2}\right)}
$$

where $\gamma=\frac{1}{a b} \int_{0}^{b} \int_{0}^{a} \Psi_{M N}^{2}(x, y) \mathrm{d} x \mathrm{~d} y$.

Let $\mathbf{F}$ be a $k \times k$ complex diagonal matrix of forces defined as $\mathbf{F}=\operatorname{diag}\left(F_{1}, F_{2}, \ldots, F_{k}\right)$. Then the velocity can be expressed as:

$$
V(x, y, \omega, t)=\frac{\omega}{2 \pi \kappa} \operatorname{trace}\left(\mathbf{F V}^{\mathbf{T}} \boldsymbol{\Omega} \boldsymbol{P}\right) \exp \left(\omega t+\frac{\pi}{2}\right)
$$

where $\kappa=2 \pi \rho_{s} a b \gamma, \mathbf{V}$ is an $M \times k$ matrix, $\mathbf{P}$ is an $N \times k$ matrix and $\Omega$ is an $M \times N$ matrix. The entries for the matrices in Equation (12) are:

$$
\begin{aligned}
V_{M k} & =\theta_{M}(x) \theta_{M}\left(x_{k}\right), P_{N k}=\zeta_{N}(x) \zeta_{N}\left(x_{k}\right), \Omega_{M N} \\
& =4 \pi^{2} /\left(\omega_{M N}^{2}-\omega^{2}\right)
\end{aligned}
$$

The method summarized here is very useful to calculate the vibrations of a clamped plate, where the velocity distribution on the plate is available, which enables fast computations of power flow.

\section{Single-stage vibration isolation system of machinery equipment}

\subsection{Derivation of force transmission}

A vibration isolation system for typical machinery equipment is shown in Figure 4, which can be proposed as a single-stage composite system shown in Figure 5.

In Figure 5, (1) denotes the machinery equipment, (2) denotes the isolators, including the stiffness and damping 


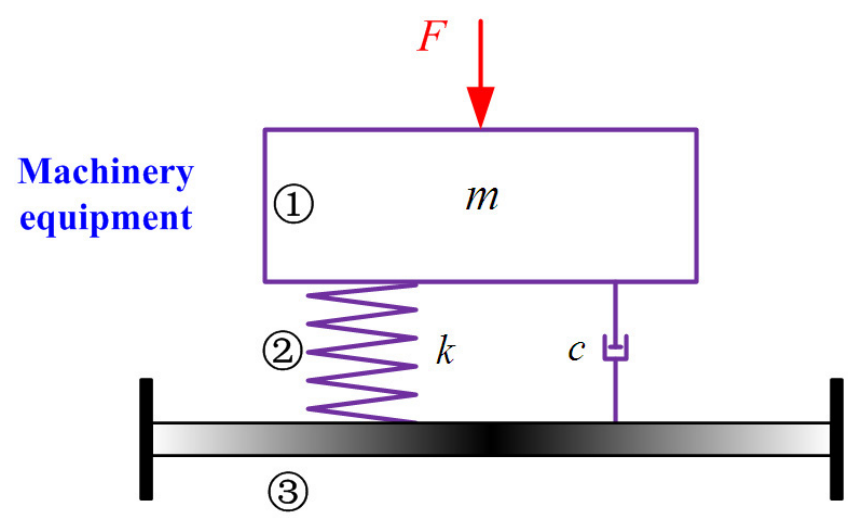

Fig. 5. Composite vibration isolation system (single-stage).

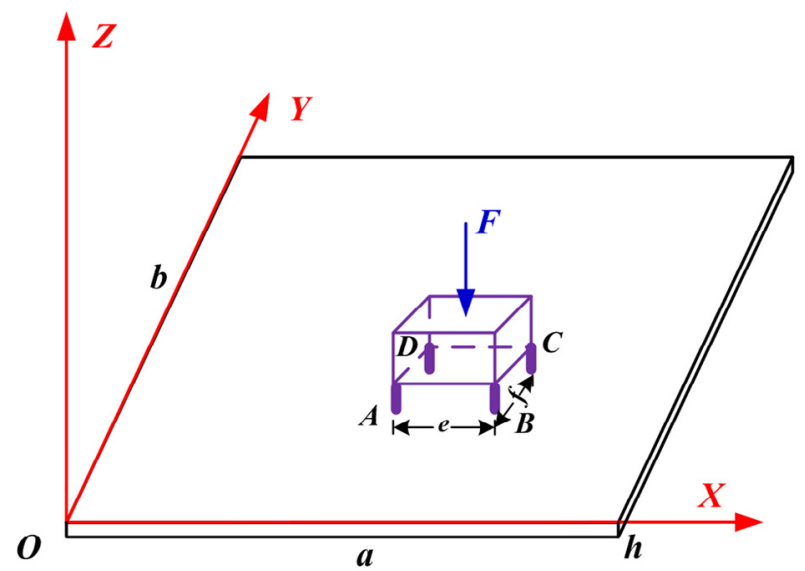

Fig. 6. Schematic diagram of a composite vibration isolation system expressed in the Cartesian coordinate system (singlestage).

components, and (3) denotes the clamped rectangular thin plate foundation. $m$ is the mass of the machinery equipment which is supported by four isolators mounted on the thin plate, and the stiffness and damping can be denoted as $k_{1 \sim 4}$ and $c_{1 \sim 4}$. The geometry of the plate is $a \times b \times h$, and for simplicity, the equipment can be considered to be a cuboid with plane size $e \times f$. The amplitude of harmonic excitation generated by the machinery equipment is F. (1) (2) (3) are considered to be a composite isolation system which can be expressed in Cartesian coordinates as shown in Figure 6, in which $\mathrm{O}$ is the coordinate origin, and $\mathrm{A}, \mathrm{B}, \mathrm{C}$ and $\mathrm{D}$ are the four corners of the equipment.

Based on the connection properties of the mechanical four-pole parameters [23], the following equation can be derived:

$$
\left[\begin{array}{l}
F_{1} \\
X_{1}
\end{array}\right]=[\hat{m}]\left[\hat{k}^{*}\right]\left[\begin{array}{l}
F_{3} \\
X_{3}
\end{array}\right]
$$

where $[\hat{m}]$ is the four-pole expression of the mass, $\left[\hat{k}^{*}\right]$ is the four-pole expression of the stiffness and damping in parallel, and $[\hat{m}]=\left[\begin{array}{cc}1 & -m \omega^{2} \\ 0 & 1\end{array}\right],\left[\hat{k}^{*}\right]=\left[\begin{array}{cc}1 & 0 \\ \frac{1}{k+i c \omega} & 1\end{array}\right]$. Then

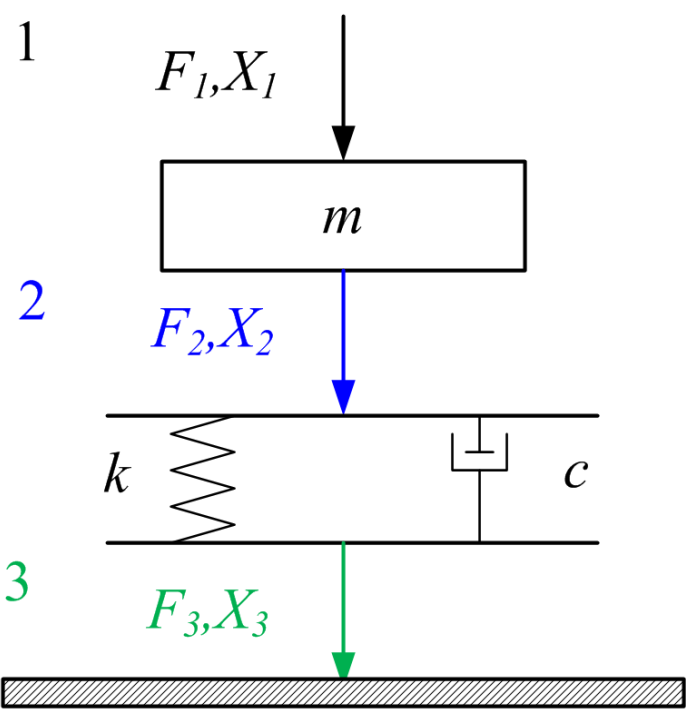

Fig. 7. Schematic diagram of the four-pole connection of the composite vibration isolation system (single-stage).

Equation (14) can be rewritten as:

$$
\begin{aligned}
{\left[\begin{array}{l}
F_{1} \\
X_{1}
\end{array}\right] } & =\left[\begin{array}{ll}
\Delta_{11} & \Delta_{12} \\
\Delta_{21} & \Delta_{22}
\end{array}\right]\left[\begin{array}{l}
F_{3} \\
X_{3}
\end{array}\right] \\
& =\left[\begin{array}{cc}
\left(1-\frac{m \omega^{2}}{k+i c \omega}\right) & -m \omega^{2} \\
\frac{1}{k+i c \omega} & 1
\end{array}\right]\left[\begin{array}{l}
F_{3} \\
X_{3}
\end{array}\right]
\end{aligned}
$$

The following formula can be easily obtained from Equation (15):

$$
F_{1}=\Delta_{11} F_{3}+\Delta_{12} X_{3}
$$

Assuming $F_{3} / X_{3}=1 / M_{\mathrm{A}}$, where $M_{\mathrm{A}}$ denotes the admittance of point A on the thin plate, Equation (16) can be rewritten by dividing by $F_{3}$ :

$$
F_{3}=\frac{1}{\Delta_{11}+\Delta_{12} \frac{X_{3}}{F_{3}}} F_{1}=\frac{1}{\Delta_{11}+\Delta_{12} M_{\mathrm{A}}} F_{1}
$$

The transmissibility is:

$$
\begin{aligned}
T_{F} & =\left|\frac{F_{3}}{F_{1}}\right| \\
& =\frac{\sqrt{k^{2}+c^{2} \omega^{2}}}{\sqrt{\left[\left(1-m \omega^{2} M_{\mathrm{A}}\right) k-m \omega^{2}\right]^{2}+c^{2} \omega^{2}\left(1-m \omega^{2} M_{\mathrm{A}}\right)^{2}}}
\end{aligned}
$$

Assuming $\omega_{0}=\sqrt{k / m}, \xi=c /\left(2 m \omega_{0}\right)$, Equation (18) can be rewritten as:

$T_{F}=\frac{\omega_{0} \sqrt{\omega_{0}^{2}+4 \xi^{2} \omega^{2}}}{\sqrt{\left[\left(1-m \omega^{2} M_{\mathrm{A}}\right) \omega_{0}^{2}-\omega^{2}\right]^{2}+4 \xi^{2} \omega^{2} \omega_{0}^{2}\left(1-m \omega^{2} M_{\mathrm{A}}\right)^{2}}}$

A variation curve of transmissibility $T_{\mathrm{F}}$ with respect to Equation (19) is shown in Figure 8, which shows that a resonant point exists. According to Equation (11), $M_{\mathrm{A}}=X\left(x_{\mathrm{A}}, y_{\mathrm{A}}\right) / F_{\mathrm{A}}, F_{\mathrm{A}}$ is the transmitted force of 


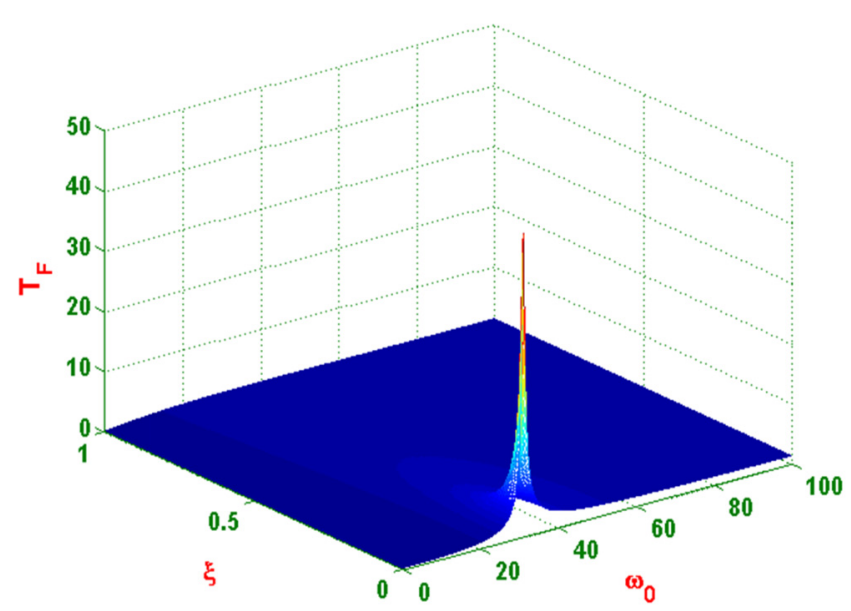

Fig. 8. Variation curve of $T_{F}-\xi-\omega_{0}$.

point A. Similarly, $M_{\mathrm{B}}, M_{\mathrm{C}}$ and $M_{\mathrm{D}}$ can be obtained. At this point, it can be assumed that there is a certain proportional relationship between the transmitted forces, $F_{\mathrm{A}}, F_{\mathrm{B}}, F_{\mathrm{C}}$ and $F_{\mathrm{D}}$, i.e. $F_{\mathrm{B}}=N_{1} F_{\mathrm{A}}, F_{\mathrm{C}}=N_{2} F_{\mathrm{A}}$, $F_{\mathrm{D}}=N_{3} F_{\mathrm{A}}$, thus $M_{\mathrm{A}}, M_{\mathrm{B}}, M_{\mathrm{C}}$ and $M_{\mathrm{D}}$ can be rewritten as,

See equation (20) next page.

\subsection{Proposed optimization strategy}

For vibrations of the plate foundation caused by machinery, the primary objective of parameter configuration is to reduce the power flow input of the four installations. The placement of equipment will certainly generate four different vibrations of the plate, and the transmitted forces and induced velocity responses of A, B, C, and D can be computed according to Section 5.1. In other words, minimization of the maximum value of the four types of power flow input can be defined as a single cost function. Meanwhile, different vibrations of the four points can negatively affect the machinery durability, and uneven vibrations will affect normal operation, and even cause paralysis. Therefore, another index is also introduced here, which seeks uniform vibration of the equipment. The two fitness functions are summarized below.

fitness1:

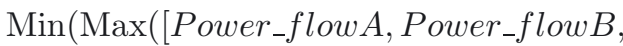
Power_flowC, Power_flowD]))

(using the form of $P L$ described in Eq. (5)) fitness2:

$\operatorname{Min}(\operatorname{var}[\operatorname{Peak}(\mathrm{A}), \operatorname{Peak}(\mathrm{B}), \operatorname{Peak}(\mathrm{C}), \operatorname{Peak}(\mathrm{D})])$ $(\operatorname{var}(\cdot)$ is a function tool based on Matlab2010, and Peak(A) denotes the vibration amplitude of A point. This fitness is converted using $\log 10$ for convenient display)

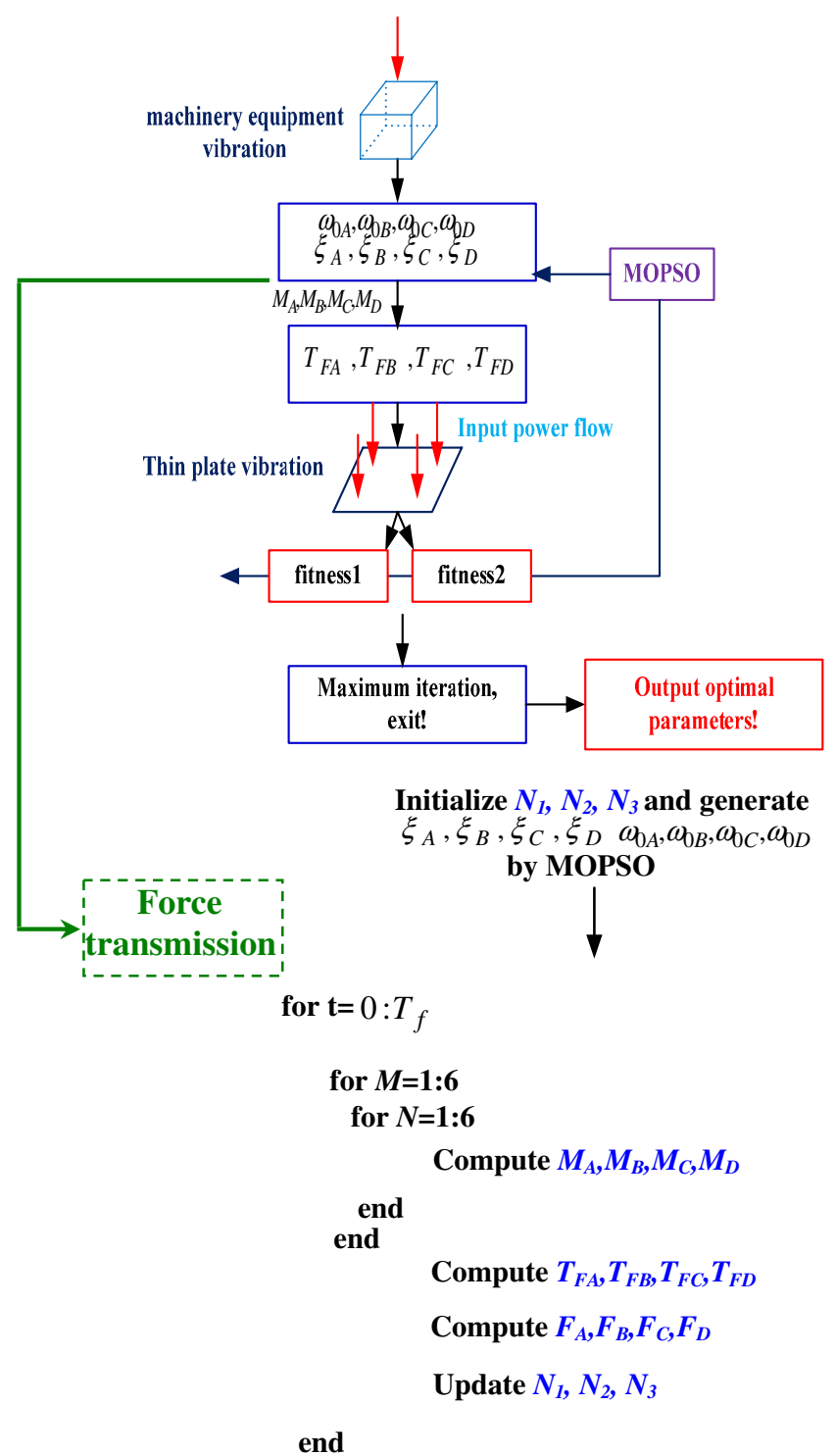

Fig. 9. Schematic chart of the multi-objective optimization strategy.

The power flow of the four installations (A, B, C and D) can be computed as discussed in the sections above. $\operatorname{var}(\cdot)$ is used to compute the variance of a vector which indicates the vibration discrepancy. The idea chart is depicted in Figure 9 .

\subsection{Numerical case 1}

Consider the thin plate foundation described in Section 4 with basic parameters:

$$
\begin{aligned}
& a=3 \mathrm{~m}, b=6 \mathrm{~m}, h=0.2 \mathrm{~m}, E=2.1 \times 10^{11}, \\
& v=0.33, \rho=7800 \mathrm{~kg} \cdot \mathrm{m}^{-3} .
\end{aligned}
$$

Discretize the thin plate to obtain the coordinates of any point $(x, y)$ on the plate, with accuracy settings denoted 


$$
\begin{aligned}
& M_{\mathrm{A}}=\frac{1}{\rho_{s} a b} \sum_{M=1}^{\infty} \sum_{N=1}^{\infty}\left\{\frac{\Psi_{M N}\left(x_{\mathrm{A}}, y_{\mathrm{A}}\right)\left[\Psi_{M N}\left(x_{\mathrm{A}}, y_{\mathrm{A}}\right)+N_{1} \Psi_{M N}\left(x_{\mathrm{B}}, y_{\mathrm{B}}\right)+N_{2} \Psi_{M N}\left(x_{\mathrm{C}}, y_{\mathrm{C}}\right)+N_{3} \Psi_{M N}\left(x_{\mathrm{D}}, y_{\mathrm{D}}\right)\right]}{\gamma\left(\omega_{M N}^{2}-\omega^{2}\right)}\right\} \\
& M_{\mathrm{B}}=\frac{1}{\rho_{s} a b} \sum_{M=1}^{\infty} \sum_{N=1}^{\infty}\left\{\frac{\Psi_{M N}\left(x_{\mathrm{B}}, y_{\mathrm{B}}\right)\left[\Psi_{M N}\left(x_{\mathrm{B}}, y_{\mathrm{B}}\right)+\frac{1}{N_{1}} \Psi_{M N}\left(x_{\mathrm{A}}, y_{\mathrm{A}}\right)+\frac{N_{2}}{N_{1}} \Psi_{M N}\left(x_{\mathrm{C}}, y_{\mathrm{C}}\right)+\frac{N_{3}}{N_{1}} \Psi_{M N}\left(x_{\mathrm{D}}, y_{\mathrm{D}}\right)\right]}{\gamma\left(\omega_{M N}^{2}-\omega^{2}\right)}\right\} \\
& M_{\mathrm{C}}=\frac{1}{\rho_{s} a b} \sum_{M=1}^{\infty} \sum_{N=1}^{\infty}\left\{\frac{\Psi_{M N}\left(x_{\mathrm{C}}, y_{\mathrm{C}}\right)\left[\Psi_{M N}\left(x_{\mathrm{C}}, y_{\mathrm{C}}\right)+\frac{1}{N_{2}} \Psi_{M N}\left(x_{\mathrm{A}}, y_{\mathrm{A}}\right)+\frac{N_{1}}{N_{2}} \Psi_{M N}\left(x_{\mathrm{B}}, y_{\mathrm{B}}\right)+\frac{N_{3}}{N_{2}} \Psi_{M N}\left(x_{\mathrm{D}}, y_{\mathrm{D}}\right)\right]}{\gamma\left(\omega_{M N}^{2}-\omega^{2}\right)}\right\} \\
& M_{\mathrm{D}}=\frac{1}{\rho_{s} a b} \sum_{M=1}^{\infty} \sum_{N=1}^{\infty}\left\{\frac{\Psi_{M N}\left(x_{\mathrm{D}}, y_{\mathrm{D}}\right)\left[\Psi_{M N}\left(x_{\mathrm{D}}, y_{\mathrm{D}}\right)+\frac{1}{N_{3}} \Psi_{M N}\left(x_{\mathrm{A}}, y_{\mathrm{A}}\right)+\frac{N_{1}}{N_{3}} \Psi_{M N}\left(x_{\mathrm{B}}, y_{\mathrm{B}}\right)+\frac{N_{2}}{N_{3}} \Psi_{M N}\left(x_{\mathrm{C}}, y_{\mathrm{C}}\right)\right]}{\gamma\left(\omega_{M N}^{2}-\omega^{2}\right)}\right\}
\end{aligned}
$$

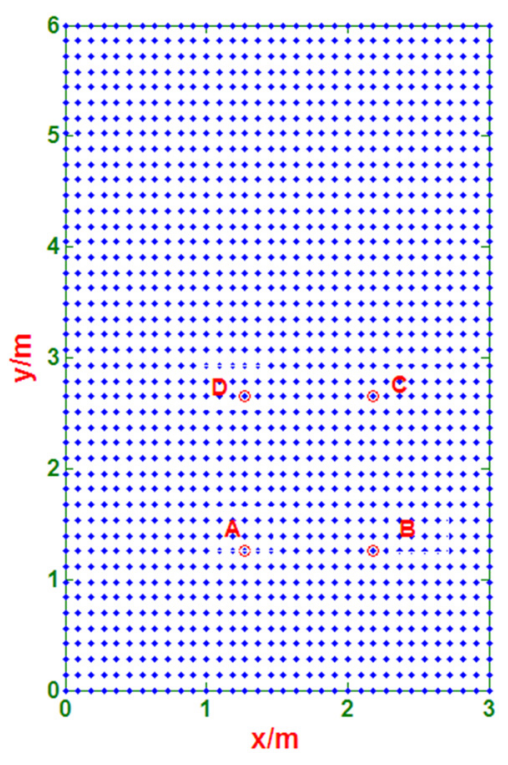

Fig. 10. Discrete grid of plate.

as $\Delta x, \Delta y$, respectively.

$$
\begin{aligned}
& \Delta x=\text { linspace }(0, a, 34), \\
& \Delta y=\operatorname{linspace}(0, b, \operatorname{round}(b / a \times 22),
\end{aligned}
$$

where linspace $(\cdot), \operatorname{round}(\cdot)$ are respectively the function tools based on Matlab2010.

The discrete grid of the plate is shown in Figure 10.

The equipment mass is assumed to be $m=200 \mathrm{~kg}$, and the plane size is $e \times f=0.90 \times 1.38 \mathrm{~m}$. The placement coordinates of point $\mathrm{A}$ are $(1.27 \mathrm{~m}, 1.26 \mathrm{~m})$; the circular frequency of the external force is $\omega=100 \pi\left(\mathrm{rad} . \mathrm{s}^{-1}\right)$, the amplitude is $1 \times 10^{3} \mathrm{~N}$, and the simulated time history is $T_{f}=1 \mathrm{~s}$. For the four isolators installed at A, B, C, and D, the natural frequencies and damping ratios, respectively, can be assumed to be:

$$
\omega_{0 A}, \omega_{0 B}, \omega_{0 C}, \omega_{0 D}, \xi_{\mathrm{A}}, \xi_{\mathrm{B}}, \xi_{\mathrm{C}}, \xi_{\mathrm{D}}
$$

The dimension is set at 8 in the MOPSO algorithm, namely $\operatorname{swarm}(i)=\omega_{0 A} \sim \omega_{0 B}, i=1 \sim 4, \operatorname{swarm}(i)=$

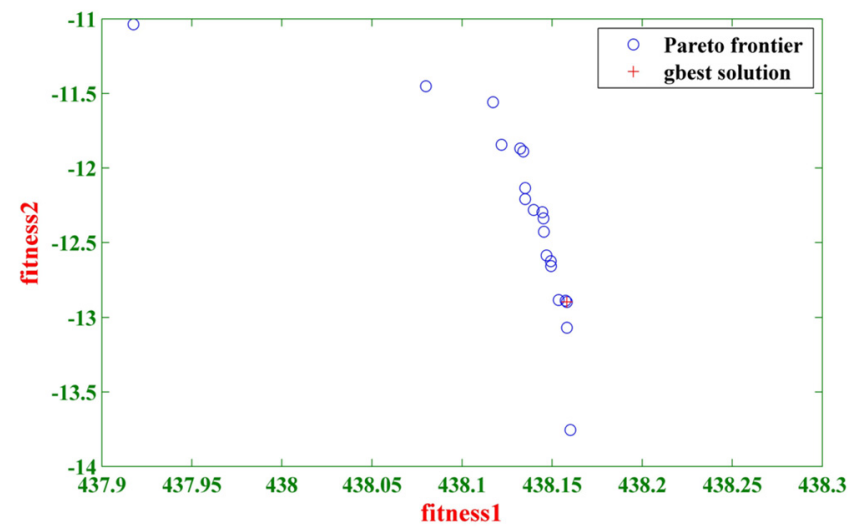

Fig. 11. Pareto frontier with gbest solution (single-stage system).

$\xi_{\mathrm{A}} \sim \xi_{\mathrm{B}}, i=5 \sim 8$, and the searching scope is arbitrarily set as

$$
\begin{aligned}
{[10,10,10,10,0.01,0.01} & , 0.01,0.01] \\
& \sim[100,100,100,100,1,1,1,1]
\end{aligned}
$$

The population size is 200; the maximum iteration is 100 ; and the learning factors are set as $c_{1}=2, c_{2}=1$. A linear changing strategy [24] is additionally used for the inertia weight factor $w$, and $w_{\max }=0.9, w_{\min }=0.4$. The Pareto frontier with gbest solution is shown in Figure 11.

The gbest solution is:

$$
\begin{gathered}
25.4017,0.0152,9.7253,0.2979,15.7525, \\
0.0147,49.6944,0.1094 \text {. }
\end{gathered}
$$

Thus, the stiffness coefficients of the four isolators can be computed as:

$$
\begin{gathered}
k_{\mathrm{A}}=3.8714 \times 10^{3} \mathrm{~N} . \mathrm{m}^{-1}, k_{\mathrm{B}}=5.6748 \times 10^{2} \mathrm{~N} . \mathrm{m}^{-1} \\
k_{\mathrm{C}}=1.4888 \times 10^{3} \mathrm{~N} . \mathrm{m}^{-1}, k_{\mathrm{D}}=1.4817 \times 10^{4} \mathrm{~N} . \mathrm{m}^{-1}
\end{gathered}
$$

and the damping coefficients of the four isolators are:

$$
\begin{aligned}
& c_{\mathrm{A}}=4.6494 \text { N.s.m }{ }^{-1}, c_{\mathrm{B}}=35.7537 \text { N.s.m }{ }^{-1} \text {, } \\
& c_{\mathrm{C}}=2.7821 \text { N.s.m }{ }^{-1}, c_{\mathrm{D}}=65.2529 \text { N.s.m }{ }^{-1} \text {. }
\end{aligned}
$$




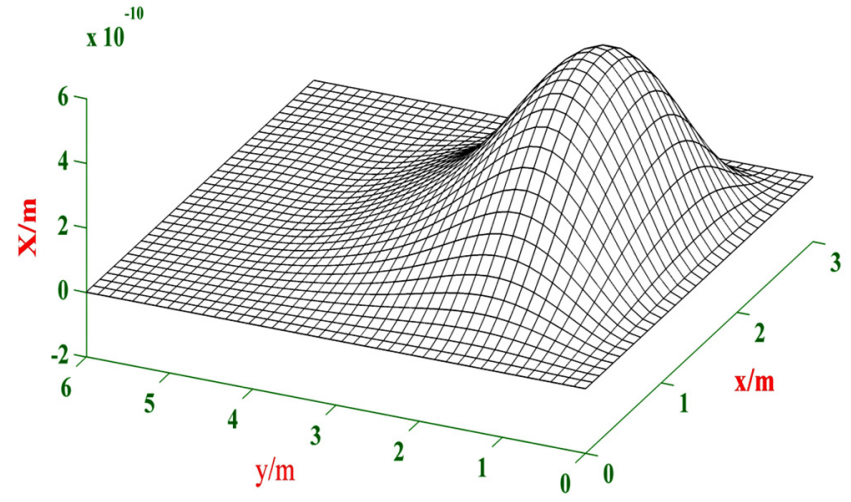

Fig. 12. Schematic diagram of the plate vibration (singlestage system).

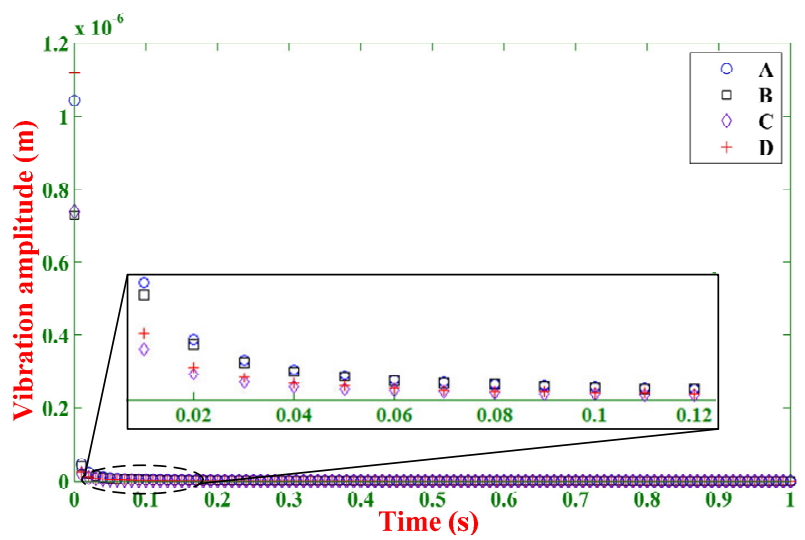

Fig. 13. Vibration amplitude of different installations after optimization (single-stage system).

The peak displacement of the plate vibration at $1.0 \mathrm{~s}$ is $5.8812 \times 10^{-10} \mathrm{~m}$, and the schematic vibration of the plate is shown in Figure 12.

The immediate variations of the amplitude with time for the four installations of A, B, C and D are listed in Figure 13, and the four amplitudes at $1.0 \mathrm{~s}$ are

$$
[4.79495,4.5563,3.0364,3.5923] \times 10^{-10} \mathrm{~m} .
$$

As can be seen from this, different vibrations tend towards a uniform vibration pattern after optimization. For other configurations, large variations in the four vibrations are seen with a huge difference even existing on the order of magnitude.

\section{Double-stage vibration isolation system for machinery equipment}

\subsection{Derivation of force transmission}

Some of the deficiencies of single-stage vibration isolation systems are overcome by resorting to double-stage systems. The effectiveness of double-stage isolation systems have been confirmed by some studies $[15,25]$. Sim-

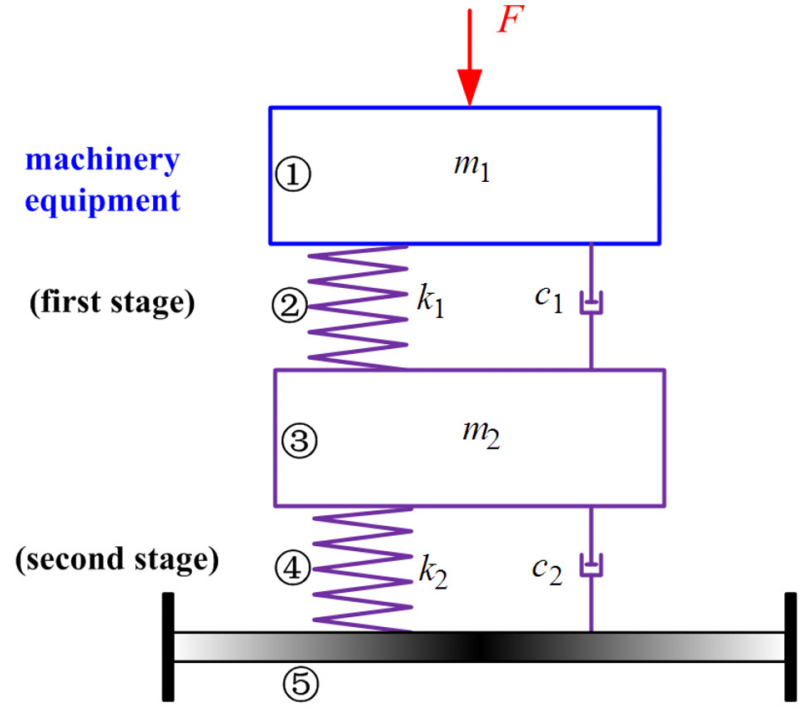

Fig. 14. Composite vibration isolation system (double-stage).

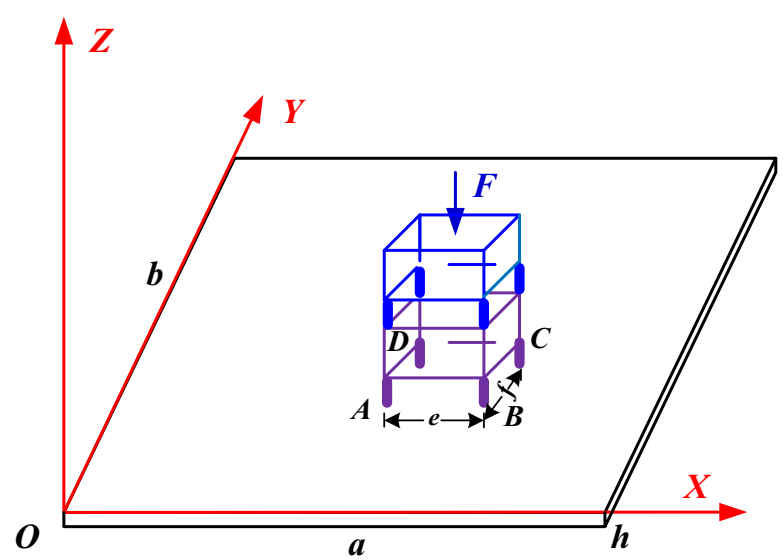

Fig. 15. Schematic diagram of the composite system expressed in Cartesian coordinate system (double-stage).

ilarly, a composite vibration isolation system is built in Figure 14.

In Figure 14, $m_{2}$ is the intermediate mass, the primary system comprises of (1) (machinery equipment) and (2) (connected isolators), the secondary system comprises of (3) (intermediate mass) and (4) (connected isolators), and (5) is the clamped thin plate. The composite system is depicted in Figure 15.

This two-stage vibration isolation system on the plate can be regarded as a tandem system consisting of two single-stage systems in series. Based on the connection properties of mechanical four-pole parameters, the following formula can be derived:

$$
\begin{aligned}
{\left[\begin{array}{l}
F_{1} \\
X_{1}
\end{array}\right] } & =\left[\begin{array}{ll}
\Delta_{11} & \Delta_{12} \\
\Delta_{21} & \Delta_{22}
\end{array}\right]\left[\begin{array}{l}
F_{\text {end }} \\
X_{\text {end }}
\end{array}\right]=\left[\begin{array}{cc}
\left(1-\frac{m_{1} \omega^{2}}{k_{1}+i c_{1} \omega}\right) & -m_{1} \omega^{2} \\
\frac{1}{k_{1}+i c_{1} \omega} & 1
\end{array}\right] \\
& \times\left[\begin{array}{cc}
\left(1-\frac{m_{2} \omega^{2}}{k_{2}+i c_{2} \omega}\right) & -m_{2} \omega^{2} \\
\frac{1}{k_{2}+i c_{2} \omega} & 1
\end{array}\right]\left[\begin{array}{l}
F_{\text {end }} \\
X_{\text {end }}
\end{array}\right]
\end{aligned}
$$




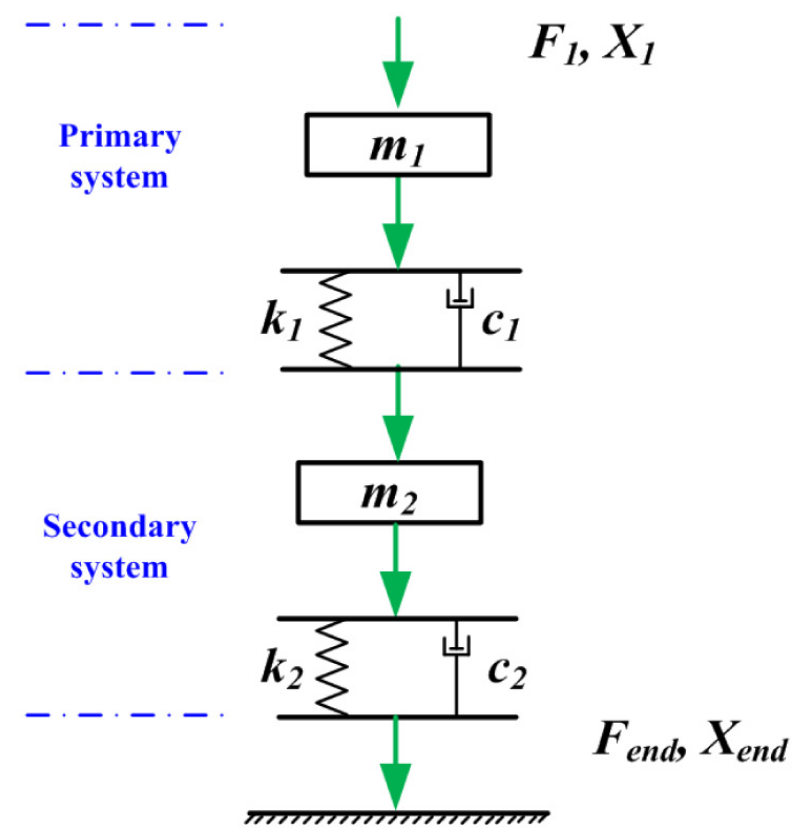

Fig. 16. Schematic diagram of four-pole connection of the composite system (double-stage).

Let $u=m_{1} / m_{2}, n_{1}=k_{1} / k_{2}, n_{2}=c_{1} / c_{2}, \omega_{n 2}=\sqrt{k_{2} / m_{2}}$, $\xi_{2}=\frac{c_{2}}{2 m_{2} \omega_{n 2}}, \theta=\frac{\omega}{\omega_{n 2}}$, thus Equation (22) can be derived as:

$$
T_{F}=1 / \sqrt{\frac{C_{1}^{2}+C_{2}^{2}}{C_{3}^{2}+C_{4}^{2}}}
$$

where

$$
\begin{aligned}
& C_{1}=A_{1} B_{3}-A_{2} B_{4}+A_{3} B_{1} M_{\mathrm{A}}+B_{1} A_{3} M_{\mathrm{A}}-B_{2} A_{4} M_{\mathrm{A}} \\
& C_{2}=A_{1} B_{4}+A_{2} B_{3}+B_{1} A_{4} M_{\mathrm{A}}+B_{2} A_{3} M_{\mathrm{A}} \\
& \text { (for the other points, } M_{\mathrm{A}} \text { can be replaced by } M_{\mathrm{B}}, M_{\mathrm{C}}, \\
& \quad \text { and } M_{\mathrm{D}} \text { ) } \\
& C_{3}=A_{3} B_{3}-A_{4} B_{4} \\
& C_{4}=A_{3} B_{4}+B_{3} A_{4} \\
& A_{1}=n_{1}-n_{2} \times\left(2 \xi_{2}\right)^{2} \theta^{2}-n_{1} \theta^{2}-u \theta^{2}+u \theta^{4}-n_{1} u \theta^{2} \\
& A_{2}=n_{1} \times 2 \xi_{2} \theta+n_{2} \times 2 \xi_{2} \theta-n_{2} \times 2 \xi_{2} \theta^{3}-u \times 2 \xi_{2} \theta^{3} \\
& A_{3}=n_{1}-n_{2} \times\left(2 \xi_{2}\right)^{2} \theta^{2} \\
& A_{4}=n_{1} \times 2 \xi_{2} \theta+n_{2} \times 2 \xi_{2} \theta \\
& B_{1}=\left(-n_{1} \omega^{2}+u \theta^{2} \omega^{2}-u n_{1} \omega^{2}\right) \frac{m_{1}}{u} \\
& B_{2}=\left(-n_{2} \times 2 \xi_{2} \omega^{2} \theta-u n_{2} \times 2 \xi_{2} \omega^{2} \theta\right) \frac{m_{1}}{u} \\
& B_{3}=n_{1} \\
& B_{4}=n_{2} \times 2 \xi_{2} \theta
\end{aligned}
$$

A slice perspective for the transmissibility described in Equation (23) with multiple parameters is shown in Figure 17.

\subsection{Numerical case 2}

The preconditions for the plate foundation and the stimulus are the same as numerical case 1 . It is worth mentioning that the plane size of the intermediate mass is assumed to be the same as the equipment. For the four isolators installed at A, B, C, and D, the parameters, respectively, can be written as:

$$
\begin{aligned}
& \xi_{2 A}, n_{1 A}, n_{2 A}, k_{2 A} \\
& \xi_{2 B}, n_{1 B}, n_{2 B}, k_{2 B} \\
& \xi_{2 C}, n_{1 C}, n_{2 C}, k_{2 C} \\
& \xi_{2 D}, n_{1 D}, n_{2 D}, k_{2 D}
\end{aligned}
$$

$u$.

The dimension is set at 17 in the MOPSO algorithm, namely $\operatorname{swarm}(i)=\xi_{2 A} \sim \xi_{2 D}, i=1 \sim 4, \operatorname{swarm}(i)=$ $n_{1 A} \sim n_{1 D}, i=5 \sim 8, \operatorname{swarm}(i)=n_{2 A} \sim n_{2 D}, i=$ $9 \sim 12, \operatorname{swarm}(i)=k_{2 A} \sim k_{2 D}, i=13 \sim 16$, and $\operatorname{swarm}(17)=u$. The searching scope is arbitrarily set as:

$$
\left[0.01,0.1,0.1,1 \times 10^{3}, 0.01,0.1,0.1,1 \times\right.
$$

$\left.10^{3}, 0.01,0.1,0.1,1 \times 10^{3}, 0.01,0.1,0.1,1 \times 10^{3}, 0.1\right] \sim$ $\left[1,100,100,1 \times 10^{6}, 1,100,100,1 \times 10^{6}, 1,100,100,1 \times\right.$ $\left.10^{6}, 1,100,100,1 \times 10^{6}, 100\right]$

The basic parameters of MOPSO are the same as the previously described case.

The Pareto frontier with the gbest solution is shown in Figure 18.

The gbest solution is

$$
\begin{gathered}
0.4823,41.9406,0.09734,1 \times 10^{3} \\
0.3880,0.09562,34.2386,1 \times 10^{3} \\
0.3266,29.5799,85.7578,1 \times 10^{3} \\
0.4742,0.09430,98.3321,1.7168 \times 105
\end{gathered}
$$

The stiffness coefficients of the isolators can then be derived as:

$$
\begin{aligned}
& k_{1 A}=4.1941 \times 10^{4} \mathrm{~N} \cdot \mathrm{m}^{-1}, k_{1 B}=95.62 \mathrm{~N} \cdot \mathrm{m}^{-1} \\
& k_{1 C}=2.9579 \times 10^{4} \mathrm{~N} \cdot \mathrm{m}^{-1}, k_{1 D}=1.6189 \times 10^{4} \mathrm{~N} \cdot \mathrm{m}^{-1} \\
& k_{2 A}=1 \times 10^{3} \mathrm{~N} \cdot \mathrm{m}^{-1}, k_{2 B}=1 \times 10^{3} \mathrm{~N} \cdot \mathrm{m}^{-1} \\
& k_{2 C}=1 \times 10^{3} \mathrm{~N} \cdot \mathrm{m}^{-1}, k_{2 D}=1.7168 \times 10^{5} \mathrm{~N} \cdot \mathrm{m}^{-1}
\end{aligned}
$$

and the damping coefficients of the isolators, respectively, are:

$$
\begin{aligned}
& c_{1 A}=1.3278 \times 10^{2} \text { N.s.m }{ }^{-1}, \\
& c_{1 B}=3.7574 \times 10^{4} \text { N.s.m }{ }^{-1}, \\
& c_{1 C}=7.9219 \times 10^{4} \text { N.s.m }{ }^{-1}, \\
& c_{1 D}=1.7280 \times 10^{6} \text { N.s.m }{ }^{-1} ; \\
& c_{2 A}=1.3641 \times 10^{3} \text { N.s.m }{ }^{-1}, \\
& c_{2 B}=1.0974 \times 10^{3} \text { N.s.m }{ }^{-1}, \\
& c_{2 C}=9.2376 \times 10^{2} \text { N.s.m }{ }^{-1}, \\
& c_{2 D}=1.7573 \times 10^{4} \text { N.s.m }{ }^{-1} .
\end{aligned}
$$




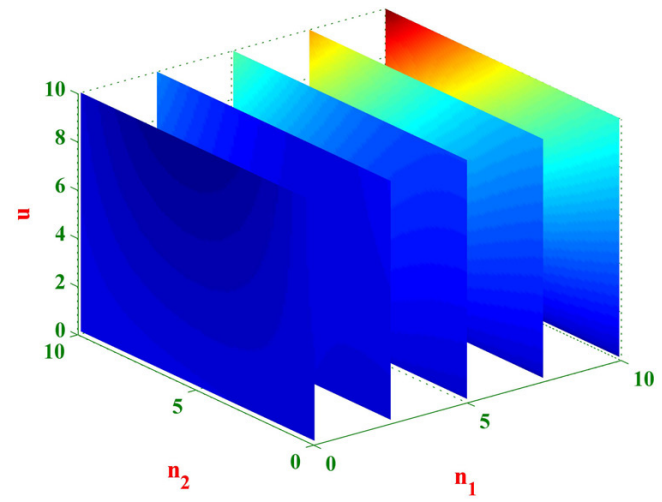

(a) slice along $n_{1}$

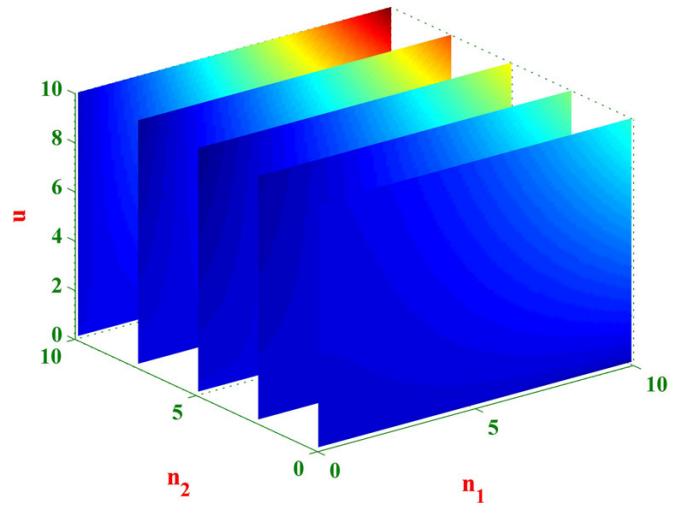

(b) slice along $n_{2}$

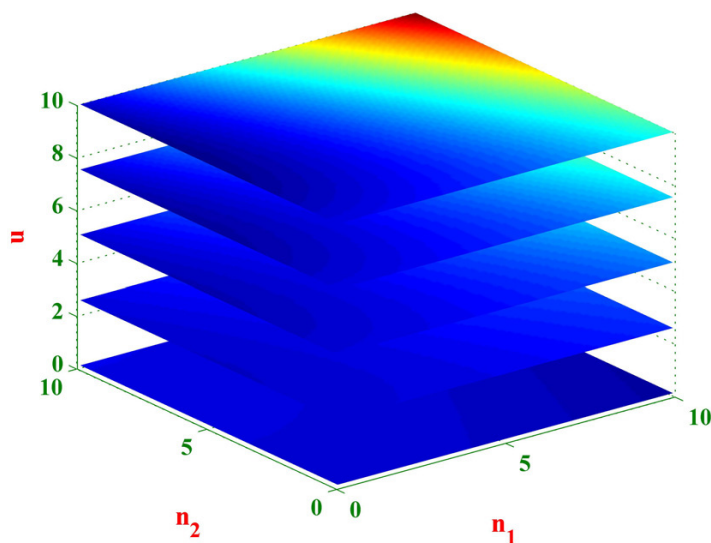

(c) slice along $\mathrm{u}$

Fig. 17. Slice plots of $T_{F}$.

The peak displacement of the plate vibration at $1.0 \mathrm{~s}$ is: $4.5701 \times 10^{-11} \mathrm{~m}$, and the plate vibration at this point is shown in Figure 19. Compared with the result for the single-stage system, there is a proportionate reduction of approximately 12 ; which indicates that the double-stage system can apparently absorb the vibrations more effectively. Similarly, the amplitude variation curves of the four installations of A, B, C and D are listed in Figure 20, and the four amplitudes at $1.0 \mathrm{~s}$ are

$$
[4.0871,3.7137,3.0023,3.4068] \times 10^{-11} \mathrm{~m} \text {. }
$$

\section{Conclusions}

In this paper, traditional vibration isolation theory is innovatively combined with the vibrations of a clamped thin plate, in order to simulate the foundation vibration forces by machinery equipment. The key intermediate variable is the point admittance on the plate. Two composite systems (single-stage and double-stage) are proposed based on the transference principle of mechanical four-pole properties, and the expressions of force transmissibility are derived.

The concept of power flow is also introduced from the perspective of energy transference, with reduction of the maximum input power flow taken as one of the fitness

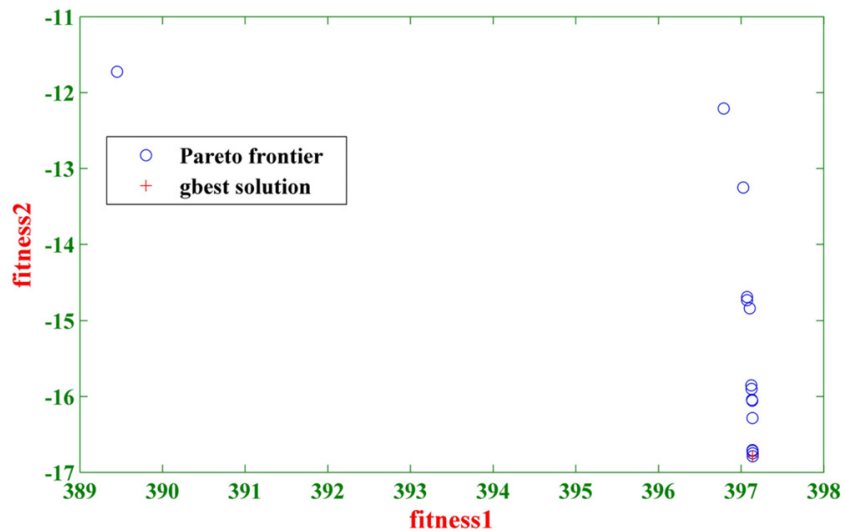

Fig. 18. Pareto frontier with gbest solution (double-stage system).

functions. It is worth mentioning that advanced vibration theory of plate vibration aptly supports this strategy. Since machinery equipment requires uniform vibrations, this is proposed as another objective for the indexes of a multi-objective problem. A novel MOPSO technique is used as the optimization tool and a global optimum gbest can be found based on Pareto domination. The numerical results show that, compared with single-stage system, the 


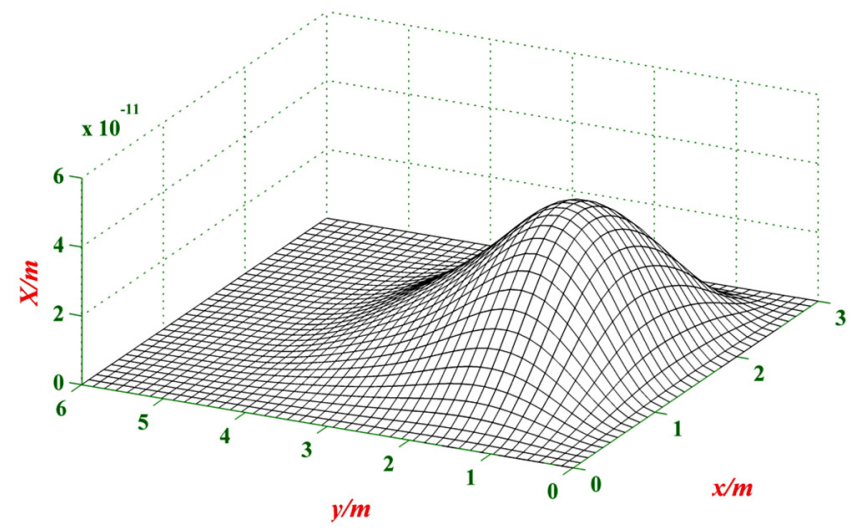

Fig. 19. Schematic diagram of the plate vibration (doublestage system).

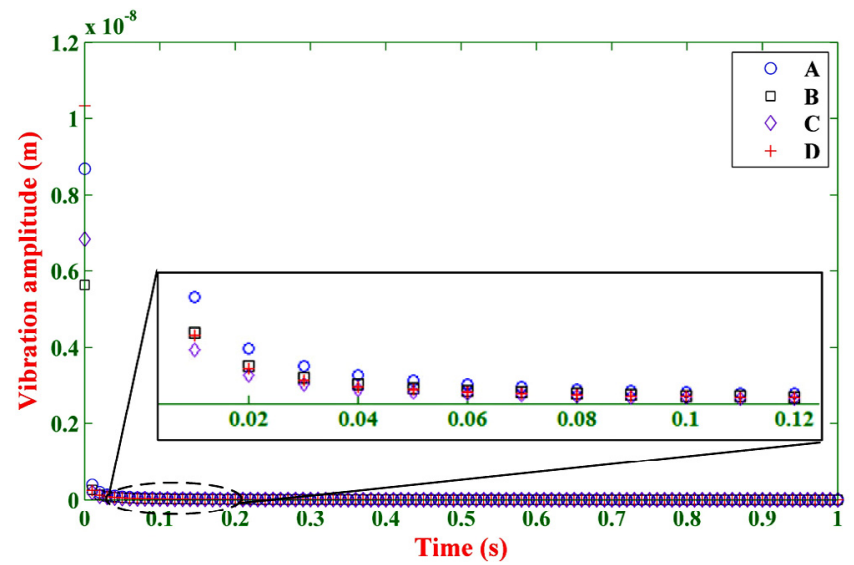

Fig. 20. Vibration amplitude of different installations after optimization (double-stage system).

double-stage system shows a more obvious and effective suppression effect.

This study challenges the traditional view of vibration isolation of machinery equipment, and gives inspiration to the use of artificial intelligence methods in combination with engineering techniques.

Acknowledgements. This research was completely supported by National Natural Science Foundation of China, and the Grants Nos. 51078123, 51179043. Valuable comments and suggestions by preparation experts of the national code "Code for vibration load design of industrial building" on the ideas carried out are gratefully acknowledged.

\section{References}

[1] M. Amabili, S. Carra, Experiments and simulations for large-amplitude vibrations of rectangular plates carrying concentrated masses, J. Sound Vib. 331 (2012) 155-166

[2] M.M. Kozupa, J.W. Wiciak, Comparison of passive and active methods for minimization of sound radiation by vibrating clamped plate, Acta Physica Polonica A 119 (2011) 1013-1017
[3] S. Li, H. Yuan, Green quasifunction method for free vibration of clamped thin plates, Acta Mechanica Solida Sinica 25 (2012) 37-45

[4] J.P. Arenas, On the vibration analysis of rectangular clamped plates using the virtual work principle, J. Sound Vib. 266 (2003) 912-918

[5] J. Yang, Y.P. Xiong, J.T. Xing, Dynamics and power flow behavior of a nonlinear vibration isolation system with a negative stiffness mechanism, J. Sound Vib. 332 (2013) 167-183

[6] S. Kumar, D.K. Chaturvedi, Optimal power flow solution using fuzzy evolutionary and swarm optimization, Int. J. Electr. Power Energy Syst. 47 (2013) 416-423

[7] K. Ayan, U. Kılıç, Artificial bee colony algorithm solution for optimal reactive power flow, Appl. Soft Comput. 12 (2012) 1477-1482

[8] H.G.D. Goyder, R.G. White, Vibrational power flow from machines into built-up structures, part I: introduction and approximate analyses of beam and plate-like foundations, J. Sound Vib. 68 (1980) 59-75

[9] H.G.D. Goyder, R.G. White, Vibrational power flow from machines into built-up structures, Part II: Wave propagation and power flow in beam-stiffened plates, J. Sound Vib. 68 (1980) 77-96

[10] H.G.D. Goyder, R.G. White, Vibrational power flow from machines into built-up structures, Part III: power flow through isolation systems, J. Sound Vib. 68 (1980) 97117

[11] J.C. Snowdon, Vibration and shock in damped mechanical systems, J. Wiley, New York-London-Sidney, 1968

[12] R.J. Pinnington, Vibrational power transmission to a seating of a vibration isolated motor, J. Sound Vib. 118 (1987) 515-530

[13] Z. Fengjiao, W. Minxiang, Multi-objective optimization of the control strategy of electric vehicle electro-hydraulic composite braking system with genetic algorithm, Adv. Mech. Eng. 7 (2015) 1-8

[14] E. Esmailzadeh, Design Synthesis of a Vehicle Suspension System Using Multi-Parameter Optimization, Vehicle System Dynamics 7 (1978) 83-96

[15] Y.D. Wei, X.B. Lai, D.Z. Chen, et al. Optimal parameters design of two-stage vibration isolation system, J. Zhejiang University 40 (2006) 893-896

[16] W. Huang, J. Xu, D.Y. Zhu, J.W. LU, Parameters Optimization of Vibration Isolation System based on Particle Swarm Optimization (PSO) Algorithm, J. Hunan University (Natural Sciences) 41 (2014) 58-66

[17] R.C. Eberhart, J. Kennedy, A new optimizer using particle swarm theory, Proceedings of the sixth international symposium on micro machine and human science, 1995, Vol. 1, pp. 39-43

[18] C.A. Coello Coello, M.S. Lechuga, MOPSO: A proposal for multiple objective particle swarm optimization, Evolutionary Computation, 2002. CEC'02. Proceedings of the 2002 Congress on. IEEE, 2002, Vol. 2, pp. 10511056

[19] C.M. Fonseca, P.J. Fleming, An overview of evolutionary algorithms in multi-objective optimization, Evol. Comput. 3 (1995) 1-16 
[20] Q.J. Wang, J.Y. Chen, H. Xu, Optimum control of complex flexible coupling system based on power flow, J. Vib. Eng. 37 (2001) 32-36

[21] K. Deb, A. Pratap, S. Agarwal, et al., A fast and elitist multi-objective genetic algorithm: NSGA-II, IEEE Trans. Evol. Comput. 6 (2002) 182-197

[22] D.E. Goldberg, J. Richardson, Genetic algorithms with sharing for multimodal function optimization, Genetic algorithms and their applications: Proceedings of the Second International Conference on Genetic NJ: Lawrence Erlbaum, Algorithms. Hillsdale, 1987, pp. 4149
[23] J.K. Yan, Mechanical vibration isolation technology, Shanghai science and technology literature press, Shanghai, 1985

[24] Y. Shi, R. Eberhart, A modified particle swarm optimizer, EEE World Congress on Computational Intelligence, 1998, pp. 69-73

[25] W.J. Ding, Vibration isolation theory, Tsinghua university press, 1998 